\title{
Covid-19 transmission, outcome and associated risk factors in cancer patients at the first month of the pandemic in a Spanish hospital in Madrid
}

\author{
J. Rogado ${ }^{1}$ (D) B. Obispo ${ }^{1} \cdot$ C. Pangua ${ }^{1} \cdot$ G. Serrano-Montero ${ }^{1} \cdot$ A. Martín Marino ${ }^{1} \cdot$ M. Pérez-Pérez ${ }^{1}$. \\ A. López-Alfonso ${ }^{1} \cdot$ P. Gullón ${ }^{2} \cdot$ M. Á. Lara ${ }^{1,3}$
}

Received: 28 April 2020 / Accepted: 9 May 2020 / Published online: 25 May 2020

(c) Federación de Sociedades Españolas de Oncología (FESEO) 2020

\begin{abstract}
Background There are no large reported series determining the Covid-19 cancer patient's characteristics. We determine whether differences exist in cumulative incidence and mortality of Covid-19 infection between cancer patients and general population in Madrid.

Material and methods We reviewed 1069 medical records of all cancer patients admitted at Oncology department between Feb 1 and April 7, 2020. We described Covid-19 cumulative incidence, treatment outcome, mortality, and associated risk factors.

Results We detected 45/1069 Covid-19 diagnoses in cancer patients vs 42,450/6,662,000 in total population $(p<0.00001)$. Mortality rate: $19 / 45$ cancer patients vs $5586 / 42,450(p=0.0001)$. Mortality was associated with older median age, adjusted by staging and histology ( 74 vs 63.5 years old, OR 1.06, $p=0.03$ ). Patients who combined hydroxychloroquine and azithromycin presented 3/18 deaths, regardless of age, staging, histology, cancer treatment and comorbidities (OR 0.02, $p=0.03$ ). Conclusion Cancer patients are vulnerable to Covid-19 with an increase in complications. Combined hydroxychloroquine and azithromycin is presented as a good treatment option.
\end{abstract}

Keywords Covid-19 $\cdot$ Cancer $\cdot$ Metastatic $\cdot$ Mortality $\cdot$ Cumulative incidence

\section{Background}

The city of Wuhan, Hubei (China), starred at December 2019 the start of a pandemic without precedents at the modern times. A novel subtype of coronavirus was discovered, with a high capacity of human-to-human transmission and an enormous virulence $[1,2]$. The novel coronavirus has a lower lethality than other previously isolated [3]; however, we are objectifying how the virus is capable of developing

J. Rogado and B. Obispo are contributed equally for this manuscript.

J. Rogado

jacobo.rogado@gmail.com

1 Medical Oncology Department, Hospital Univeristario Infanta Leonor, Gran vía del este, 81, 28031 Madrid, Spain

2 Public Health and Epidemiology Research Group, Universidad de Alcalá, Alcalá de Henares, Spain

3 Universidad Complutense de Madrid, Madrid, Spain serious unilateral and bilateral pneumonias and inflammatory responses [4].

The arrival of the virus at Spain took place on January 31 st, becoming in one of the most worrying active focus in Europe since early March. A few days later, an intracommunity transmission was detected, declaring the health alert and focusing all the hospital cares on Covid-19 patients.

Currently, Spain, and the region of Madrid is one of the most affected places in the entire world. Cancer patients should continue their diagnoses and treatments due to avoid complications of their previous diseases, as suggested by Cortiula and Curigliano [5, 6]. Their visits should be maintained in all Medical and Radiation Oncology departments, with the consequent increased risk of Covid-19. In a Chinese series, an increase in Covid-19 incidence in oncology population was described, attributing it to the maintenance of visits to the hospital without performing any Covid-19 screening [7]. Another recent study described an increase in more serious cases in cancer patients due to their acquired immunosuppression status, especially those who are receiving 
specific treatment [8]. However, another report described similar characteristics and infection rate, but they detected most infected patients were cancer survivors, without active cancer disease or any recent specific treatment received [9].

In our study, we determine whether exists differences in cumulative incidence and mortality of Covid-19 between cancer patients visiting our Medical Oncology department and general population, in the current epicenter of the pandemic in Europe (Madrid, Spain). We also describe clinical and demographic factors associated with poor prognosis and Covid-19 treatment outcomes in a cohort of cancer patients where an advanced stage predominates.

\section{Methods}

We retrospectively review 1069 medical records admitted to Medical Oncology department at Hospital Universitario Infanta Leonor of Madrid, from February 1, 2020, to April 7, 2020. We study cumulative incidence of Covid-19 infection and its mortality in cancer patients.

Covid-19 diagnosis was made based on WHO criteria and/or confirmed by RT-PCR of nasopharyngeal specimens. Severe Covid-19 infection was defined as presence of bilateral pneumonia with CURB-65 scale score $\geq 2 / \mathrm{FiO}_{2} \geq 35 \%$ or admission to an Intensive Care Unit (ICU).

Descriptive analyses are reported as relative frequencies for discrete variables. Continuous variables are reported as mean \pm standard deviation (SD) or median and interquartile range (IQR) for normal and not normally distributed variables, respectively. To determine differences on mortality between cancer patients and general population, Fisher's Exact Test were performed. To determine the relationship between clinical and demographic risk factors with mortality, Chi square Test, univariate logistic regression and multivariate logistic regression were performed. Statistical analyses were carried out with STATA SE version 14.1 (StataCorp, College Station, TX, USA). A $p$ value $<0.05$ was considered statistically significant.

\section{Results}

Forty-five patients were diagnosed with Covid-19 from February 1, 2020, to April 7, 2020, within 1069 cancer patients (4.2\%) visited at Medical Oncology department in Hospital Universitario Infanta Leonor in Madrid (Spain). The median follow-up until data cut off was 14 days (range 1-28). The median age was 71 years old (range 34-90). Regarding sex, a higher prevalence of males were detected $(n=30,66.7 \%)$. Most patients had metastatic disease $(n=26,57.8 \%)$. Most frequent histology were lung cancer $(n=17,37.8 \%)$. The overall survivals were similar between patients with active treatment or not active treatment ( 8 months vs 7 months). All patients who were able to receive treatment for Covid19 infection received hydroxychloroquine $(n=37,82.2 \%)$, most combined with lopinavir/ritonavir $(n=14,31.1 \%)$ or azithromycin $(n=18,40.0 \%)$. All patients required admission except for 7 patients (84.5\%), 29 of them were diagnosed with severe Covid-19 infection (64.4\%). All clinical and demographic characteristics, comorbidities and treatment description are shown in Table 1.

The cumulative incidence in our cohort was higher (4.2\%) than detected in Madrid: 42,450 over 6,662,000 population at data cut off [10] $(0.63 \%, p<0.00001)$. Mortality in cancer patients it was amounted to 19 of 45 patients $(42.2 \%)$, compared to observed in general population, with 5586 deaths among 42,450 Covid-19 patients [9] $(13.2 \%, p=0.0001)$ and in our hospital, with 1878 covid-19 patients admitted and 192 deaths $(10.2 \%, p=0.0001)$.

Univariate analyses with differences in clinical and demographic characteristics, comorbidities, Covid-19 symptoms and treatment outcome between survivors and dead's patients are shown in Table 2.

In multivariate analysis, we detected that dead's patients had an older age than survivors (74 versus 63.5 years old), regardless of histology and staging (OR 1.06, CI 1.00-1.12, $p=0.03$ ). Combined hydroxychloroquine and azithromycin treatment showed a better outcome, with only 3 deaths among 18 patients under this treatment (12.5\%), adjusted by median age, histology, staging, cancer treatment received and hypertension (OR 0.02, CI 0.01-0.73, $p=0.03$ ).

\section{Discussion}

In our study, we obtained a cumulative incidence of Covid19 in cancer patients higher than our general population [10]. However, considering the characteristics of our patients, their specific treatments and state of immunosuppression, it is expected to observe a higher incidence of cases. Furthermore, we know that these patients visit frequently the hospital, establishing an added risk of Covid-19.

Our general population had a high fatality rate, with a $13.2 \%$ of deaths at data cut off [9]. However, we obtained significant differences within mortality terms in cancer patients, rising up to $42.2 \%(p=0.0001)$. When we searched differences in the mortality rate between all Covid-19 patients admitted to our center and our Covid-19 cancer population, we also observed differences, with a mortality rate in our hospital of $9.8 \%$ in all Covid-19 patients $(p=0.0001)$.

We believe that our patients could be acquiring nosocomial transmission, as suggested by Yu et al. [7] with up to twice the incidence of cases with respect to general population in China (OR 2.31, CI 1.89-3.02), as described also by Zhang et al. [8] and by Liang et al. [10] with an incidence 
Table 1 Clinical and demographic characteristics, outcome, symptoms and treatment of Covid-19 cancer patients

\begin{tabular}{|c|c|c|}
\hline Characteristics & $N$ & Percentage $(\%)$ \\
\hline \multicolumn{3}{|l|}{ Sex } \\
\hline Male & 30 & 66.7 \\
\hline Female & 15 & 33.3 \\
\hline \multicolumn{3}{|l|}{ Cancer type } \\
\hline Lung cancer & 17 & 37.8 \\
\hline Breast cancer & 6 & 13.3 \\
\hline Colorectal cancer & 6 & 13.3 \\
\hline Prostate cancer & 5 & 11.1 \\
\hline Other & 11 & 24.5 \\
\hline \multicolumn{3}{|l|}{ Cancer staging } \\
\hline Localized & 6 & 13.3 \\
\hline Locally advanced & 13 & 28.9 \\
\hline Metastatic & 26 & 57.8 \\
\hline \multicolumn{3}{|l|}{ Cancer treatment } \\
\hline No active treatment & 13 & 28.9 \\
\hline Chemotherapy & 19 & 42.2 \\
\hline TKIs & 2 & 4.4 \\
\hline Immunotherapy & 1 & 2.2 \\
\hline New diagnosis & 2 & 4.4 \\
\hline Others & 8 & 17.9 \\
\hline \multicolumn{3}{|l|}{ Comorbidities } \\
\hline Hypertension & 23 & 51.1 \\
\hline Diabetes mellitus & 13 & 28.9 \\
\hline Cardiovascular disease & 4 & 8.8 \\
\hline Chronic kidney disease & 3 & 6.7 \\
\hline COPD & 13 & 28.9 \\
\hline Obesity & 6 & 13.3 \\
\hline \multicolumn{3}{|l|}{ Covid-19 diagnosis } \\
\hline Hospitalization admission & 38 & 84.4 \\
\hline ICU admission & 0 & 0 \\
\hline Home management (discharged from hospital at diagnosis) & 7 & 15.6 \\
\hline \multicolumn{3}{|l|}{ Covid-19 outcome } \\
\hline Dead & 19 & 42.2 \\
\hline Alive & 26 & 57.8 \\
\hline Severe covid-19 infection & 29 & 64.4 \\
\hline \multicolumn{3}{|l|}{ Symptoms } \\
\hline Fever & 38 & 84.4 \\
\hline Cough & 39 & 86.7 \\
\hline Myalgia & 10 & 22.2 \\
\hline Dyspnea & 38 & 84.4 \\
\hline Diarrhea & 1 & 2.2 \\
\hline \multicolumn{3}{|l|}{ Covid-19 treatment } \\
\hline Lopinavir/ritonavir + hydroxychloroquine & 10 & 22.2 \\
\hline Hydroxychloroquine + azithromycin & 18 & 40.0 \\
\hline Lopinavir/ritonavir + hydroxychloroquine + azithromycin & 4 & 8.8 \\
\hline Hydroxichloroquine & 2 & 4.4 \\
\hline Hydroxychloroquine + azithromycin + steroids & 2 & 4.4 \\
\hline Hydroxychloroquine + azithromycin + steroids + tocilizumab & 1 & 2.2 \\
\hline
\end{tabular}

TKIs tyrosine kinase inhibitors, COPD chronic obstructive pulmonary disease 
Table 2 Clinical and demographic parameters in both subgroup of Covid-19 cancer patients (dead's patients or survivors)

\begin{tabular}{|c|c|c|c|}
\hline & $\begin{array}{l}\text { Survivor patients } \\
(N=26)\end{array}$ & Dead patients $(N=19)$ & $p$ value \\
\hline Age, median & 74 & 63.5 & 0.01 \\
\hline Male, $n(\%)$ & $16(61.5)$ & $14(73.68)$ & 0.39 \\
\hline \multicolumn{4}{|l|}{ Cancer characteristics } \\
\hline \multicolumn{4}{|l|}{ Histology } \\
\hline Lung cancer, $n(\%)$ & $8(30.8 \%)$ & $9(47.3 \%)$ & 0.20 \\
\hline \multicolumn{4}{|l|}{ Cancer treatment } \\
\hline Chemotherapy, $n(\%)$ & $13(50.0 \%)$ & $6(31.5 \%)$ & 0.44 \\
\hline \multicolumn{4}{|l|}{ Staging } \\
\hline Metastatic disease, $n(\%)$ & $13(50.0 \%)$ & $13(68.4 \%)$ & 0.53 \\
\hline \multicolumn{4}{|l|}{ Covid-19 characteristics } \\
\hline \multicolumn{4}{|l|}{ Covid-19 treatment } \\
\hline $\begin{array}{l}\text { Hydroxychloroquine + azithromycin, } \\
n(\%)\end{array}$ & $15(57.7 \%)$ & $3(15.7 \%)$ & 0.008 \\
\hline \multicolumn{4}{|l|}{ Covid-19 symptoms } \\
\hline Fever, $n(\%)$ & $23(88.5 \%)$ & $15(78.9 \%)$ & 0.38 \\
\hline Cough, $n(\%)$ & $23(88.5 \%)$ & $16(84.2)$ & 0.67 \\
\hline Severe infection, $n(\%)$ & $12(46.1 \%)$ & $17(89.5 \%)$ & 0.003 \\
\hline \multicolumn{4}{|l|}{ Comorbidities } \\
\hline Hypertension, $n(\%)$ & $11(42.3 \%)$ & $12(63.1 \%)$ & 0.16 \\
\hline \multicolumn{4}{|l|}{ Diabetes, $n(\%)$} \\
\hline COPD, $n(\%)$ & $8(30.7 \%)$ & $5(26.3 \%)$ & 0.74 \\
\hline $\mathrm{CKD}, n(\%)$ & $4(15.3 \%)$ & $2(10.5 \%)$ & 0.63 \\
\hline Cardiovascular disease, $n(\%)$ & $2(7.7 \%)$ & $1(5.3 \%)$ & 0.74 \\
\hline Obesity, $n(\%)$ & $4(15.3 \%)$ & $2(10.5 \%)$ & 0.63 \\
\hline
\end{tabular}

$C O P D$ chronic obstructive pulmonary disease, $C K D$ chronic kidney disease of $1 \%$ in cancer patients versus $0.29 \%$ in general population in China. We also detected in our study a higher cumulative incidence as in our general population $(4.3 \%$ vs $0.63 \%$, $p<0.00001$ ).

In two of these studies, they observed that Covid-19 presented a higher lethality in cancer population [8, 9]. Zhang et al. [8] report a mortality of $28.6 \%$ and a severe event rate of $53.6 \%$, as in the study completed by Liang et al. [9], where they described a rate of complications and mortality $(39 \%)$ higher than the rate detected in all the infected patients in their hospital (8\%).

In both studies, the mortality rate is lower than our report. We found a mortality rate in our study of $42.2 \%$ of cancer patients. These facts are probably related because the authors included a large cohort of cancer survivors [9], or their cancer staging was not predominantly metastatic [8].

In previously reported studies, it is described that chemotherapy is a risk factor in cancer patients with Covid-19 [6-8]. However, we did not detect an increase in mortality in patients who were receiving this treatment in our cohort. Chemotherapy could be contributing to decrease the inflammation that has been associated with a higher mortality in Covid-19 [4].
Finally, we observed that those cancer patients treated with combined hydroxychloroquine and azithromycin had a better outcome. This fact are not mentioned in the rest of the articles, either due to the absence of data collection $[7,9]$ or because the drugs outcome is not reported [8]. This is at the moment, to our knowledge, the only report of Covid-19 disease in cancer patients in Europe and the largest series reported including oncology patients.

\section{Conclusion}

Covid-19 triggers serious complications in cancer patients, so our efforts should be made to reduce visits to hospital during the pandemic. The severity of the infection at admission and the elderly patients are independents indicators of mortality, and combined treatment with hydroxychloroquine and azithromycin seems a good option in cancer patients.

Acknowledgements The authors express their gratitude to the patients and their families for participation in this study. 
Author contributions JR contributed to the conception and design of the study, data acquisition, statistical analysis, interpretation of the data and writing of the manuscript. BO contributed to the conception and design of the study, interpretation of the data and writing of the manuscript. CP, GS-M, AMM, MP-P, AL-A contributed to the acquisition of the data. PG contributed to the statistical analysis and interpretation of the data. MAL contributed to the conception and design of the study, interpretation of the data and writing of the manuscript. All authors reviewed and approved the final version of the manuscript.

Funding No funding required.

\section{Compliance with ethical standards}

Conflict of interest The authors declare no conflict of interest for the present work.

Ethical approval The study was approved by the Ethical Committee of our hospital (code: COVID-CANCER-HUIL).

Informed consent The study meets all requirements for informed consent exemption according to the "International Ethical Guidelines for Health-related Research Involving Humans” (CIOMS-WHO 2016).

\section{References}

1. Wang C, Horby PW, Hayden FG, et al. A novel coronavirus outbreak of global health concern. Lancet. 2020;395(10223):470-3. https://doi.org/10.1016/S0140-6736(20)30185-9.

2. Li Q, Guan X, Wu P, et al. Early transmission dynamics in Wuhan, China, of novel coronavirus-infected pneumonia. N Engl J Med. 2020;382:1199-207.
3. Yin Y, Wunderink RG. MERS, SARS and other coronaviruses as causes of pneumonia. Respirology. 2018;23(2):130-7.

4. Conti P, Ronconi G, Caraffa A, et al. Induction of pro-inflammatory cytokines (IL-1 and IL-6) and lung inflammation by Coronavirus-19 (COVI-19 or SARS-CoV-2): anti-inflammatory strategies. J Biol Regul Homeost Agents. 2020;34(2):1.

5. Cortiula F, Pettke A, Bastoletti M, et al. Managing COVID-19 in the oncology clinic and avoiding the distraction effect. Ann Oncol. 2020;31(5):553-5. https://doi.org/10.1016/j.annonc.2020.03.286.

6. Curigliano G. How to guarantee the best of care to patients with cancer during the COVID-19 epidemic: the Italian experience. Oncologist. 2020. https://doi.org/10.1634/theoncolog ist.2020-0267.

7. Yu J, Ouyang W, Chua MLK, et al. SARS-CoV-2 transmission in patients with cancer at a tertiary care hospital in Wuhan, China. JAMA Oncol. 2020. https://doi.org/10.1001/jamao ncol.2020.0980.

8. Zhang L, Zhu F, Xie C, et al. Clinical characteristics of COVID19-infected cancer patients: a retrospective case study in three hospitals within Wuhan, China. Ann Oncol. 2020. https://doi. org/10.1016/j.annonc.2020.03.296.

9. Liang W, Guan W, Chen R, et al. Cancer patients in SARSCoV-2 infection: a nationwide analysis in China. Lancet Oncol. 2020;21(3):335-7. https://doi.org/10.1016/S1470-2045(20)30096 $-6$.

10. Informe no 21. Situación de COVID-19 en España a 6 de abril de 2020. Equipo COVID-19. RENAVE. CNE. CNM (ISCIII)

Publisher's Note Springer Nature remains neutral with regard to jurisdictional claims in published maps and institutional affiliations. 